\title{
Perception of Tinnitus Handicap And Stress Across Age Groups in Normal Hearing
}

Anuradh Sharma ${ }^{1}$

Nilanshu sood ${ }^{2}$

Sanjay Munjal ${ }^{3}$

Naresh Panda ${ }^{4}$

\begin{abstract}
Introduction: This research was conducted to investigate the severity of tinnitus, its impact on subject's daily, personal and emotional life, which varies widely across the age groups.

Methodology: Sample population of 60 unilateral tinnitus subjects with normal hearing between the age ranges of 15-55years were divided into four age groups. Tinnitus severity was measured using tinnitus severity index, impact of tinnitus on daily life was documented through tinnitus handicap inventory and the stress levels through perceived stress scale.

Results: A high frequency tinnitus was observed in age group of 56-65years, in contrast to noise like tinnitus in subjects aged 15-25years. The tinnitus was found to be most handicapping (38-56; moderate handicap) in Group 4(56-65years), the perceived stress levels were also falling in very high range (21 and over). Tinnitus severity index did not show any significant difference between the Group 1:15-25yrs, Group 2: 26-40yrs, Group 3:41-55yrs, and however group 4(56-65 yrs) did report with mild severity. The outcomes of present study demonstrated that elderly subjects certainly require modifications in the test protocols and referrals to address to their significant responses to tinnitus.
\end{abstract}

Keywords: Tinnitus, Tinnitus handicap inventory, perceived stress scale, tinnitus severity scale.

${ }^{1}$ Department of Otorhinolaryngology Speech and Hearing Chandigarh. Institute of Medical Education and Research, Chandigarh

${ }^{2}$ Department of Otolaryngology, Speech and hearing unit, Institute of Medical Education and Research, Chandigarh

${ }^{3}$ Department of Otolaryngology Speech and Hearing Unit, Institute of Medical Education and Research, Chandigarh., India

${ }^{4}$ Department of Otolaryngology, Institute of Medical Education and Research, Chandigarh, India

Department of Otorhinolaryngology- Speech and Hearing Chandigarh. Institute of Medical Education and Research, Chandigarh, India, E-mail: anuradha2ks@yahoo. com Phone: +09872987304

Paper submitted on December 17, 2019; and Accepted on December 08, 2020 


\section{INTRODUCTION}

A phantom sound not associated with any external stimulus is known as tinnitus, $33 \%$ of the elderly population are affected by tinnitus ${ }^{1,2}$. The prevalence of tinnitus has been estimated at 10 to $15 \%$ based on data obtained from epidemiologic studies conducted in different countries, ${ }^{3}$. Few studies report tinnitus is more prevalent among men, but is variable across various age groups ${ }^{4}$. The prevalence of chronic tinnitus increases with increasing age, peaking at $14.3 \%$ in people between 60 and 69 years of age, 5 . Researchers postulate that changes in the neuroplastic potential across the life span play a critical role in tinnitus generation. During senescence, the neuroplastic changes are predominant; hence, it influences not only the incidence of tinnitus but also distress related to tinnitus ${ }^{6}$. Tinnitus perception has also been strongly correlated with emotional impact. A large number of tinnitus individuals can cope with it, only 1 in 5 are reported to have emotional symptoms, ${ }^{7}, 0.5 \%$ of them are so severely impaired that it hampers their day to day functioning ${ }^{8}$. A recent study reported that "tinnitus can lead to significant distress, depression, anxiety and a decrease in quality of life" 9 . Tinnitus becomes more severe with stress, \& the frequency of its occurrence increases in the elderly, ${ }^{10}$. However, many researchers have proposed that annoyance perceived due to tinnitus is possibly associated with a person's psychological state rather than hearing thresholds, It is complicated to understand why only a few tinnitus patients report it to be disabling and handicapping. Furthermore, the distress and severity of tinnitus vary widely across age groups contributing to poor understanding of factors related to tinnitus. It is very important to deduce the severity of tinnitus across age groups for health care implications, as well. Primarily an accurate estimate of the severity of tinnitus is required for appropriate resource allocation by governing authorities for the treatment of such disorders. Secondly, it helps us in making accurate clinical decisions regarding the tinnitus population, ${ }^{11,12}$. For these reasons, there is a requirement for further investigation of the impact of tinnitus among different age groups, as the studies conducted till now are very less in number, and none of them completely explain the severity, handicap, or stress variation among different age groups. Understanding these parameters would help us facilitate treatment and lead to better patient morbidity. Hence, the objectives of the present study were, to examine the variations in pitch and loudness of tinnitus among different age groups, further to evaluate the association between the severity of tinnitus and different age groups on the tinnitus severity Index, tinnitus distress on the perceived stress scale and impact on daily life through tinnitus handicap inventory.

\section{METHODS}

A total of 60 subjects having unilateral tinnitus (either in the right or left ear), who visited the tertiary care hospital were enrolled in the study. Participants reporting with any history of health diseases, psychiatric illness, and systemic disorder, or any tinnitus-related pathologies were excluded from the study. The participants were divided into four groups (15 in each group) based on their age range, Group 1: age range-15-25, Group 2: age range-26-40, Group 3: age range-41-55, Group 4: age range-56-65. All the subjects were administered with a history questionnaire and routine audiometric evaluation.

Pure tone audiometry: All the participants underwent pure tone audiometry on Madsen Orbiter 922 clinical audiometer using TDH-39 headphones. Pure tone thresholds were recorded at $250 \mathrm{~Hz}, 500 \mathrm{~Hz}, 1 \mathrm{KHz}, 2 \mathrm{KHz}, 4 \mathrm{kHz}$, and $8 \mathrm{KHz}$. Pure tone average was taken for frequencies $500 \mathrm{~Hz}, 1 \mathrm{KHz}$, $2 \mathrm{KHz}$, and $4 \mathrm{KHz}$, pure tone average below $25 \mathrm{dBHL}$ was considered as normal hearing.

\section{Demographics}

A detailed case history evaluation was carried out, followed by measurement of tinnitus frequency and loudness. The contralateral ear to the tinnitus was used for conducting the tinnitus matching. For tinnitus matching pure tone was presented at $1 \mathrm{KHz}$ and $10 \mathrm{~dB}$ above the patient's threshold in that ear. The pitch was changed till the closest match to the pitch of their tinnitus was obtained. After the frequency match, the stimulus was presented (pure tone or noise) $10 \mathrm{dBHL}$ below the hearing threshold of the subject. The intensity was then increased in $2 \mathrm{~dB}$ steps until the subject indicated that a perfect match was attained. All the subjects were assessed using a systematic interview and observation protocol for assessment of tinnitus-related distress, handicap, and severity. Effects of tinnitus on hearing, lifestyle, general health, and emotional disturbances like despair or frustration were recorded through Questionnaires: Tinnitus severity index (TSI), Tinnitus handicap inventory (THI), Perceived Stress Scale (PSS). An adapted Hindi version of THI was administered to all the patients, following the administration of the tinnitus handicap questionnaire, total scores were calculated, 13-15. The responses of subjects were scored based on three options; yes, no and sometimes ${ }^{16,17}$. The questions with the response 'yes' were given a score of 4 , whereas two were given for 'some time,' and ' 0 ' was given to 'no' response. The tinnitus handicap was then graded as mild 18, moderate (38-56) and severe (58-76) according to tinnitus handicap inventory. The stress level was rated as low (8-11), average (12-15), high (16-20) and very high (21 \& over) based on scores obtained on PSS. Tinnitus severity was rated based on 12 questions, and rating of 0 to 5 was given, scores of 1-12(very mild), 13-24 (mild), 2536(moderate), 37-48 (severe), 49-60 (catastrophic).

Statistical analysis: SPSS version 21 windows software was used for analysis. The descriptive statistics were used to obtain mean, the standard deviation for continuous variables and frequency and percentages for the discontinuous variables. The intergroup results were compared using MANOVA, and post hoc analysis was carried out. An Independent t-test was used to examine the statistical significance $(P<0.05)$. 


\section{RESULTS}

The age ranges taken for study and mean age of subjects and other demographic variables have been displayed in Table 1. Subjects of Group1 (15-25years) showed significant differences from Group 4 (56-65years) in terms of tinnitus frequency as well as loudness. Group 4 subjects perceived the pitch of tinnitus as tonal (93.3\% subjects); however, the majority (86.6\%) of group 1 reported tinnitus, as noisy. Subjects in group 4 had tinnitus lasting for more than 12 months.

The mean and standard deviation values among the three groups are shown in Table 2. In applying the ANOVA test, significant differences were observed among all the four groups on tinnitus handicap inventory, perceived stress scale as well as on tinnitus severity index.

The multiple comparisons were made for the tinnitus frequency among the four age groups. The significant difference was only observed between group 1 and group $4(P=0.025)$, as shown in Table 3 , indicating that subjects in the age range 15-25 had a different frequency of tinnitus in comparison to the subjects in the age range of 55-60yrs.
Post Hoc test results of tinnitus handicap scores showed significant differences between Group 1 and Group 2 $(P=0.021)$, Group 1 and Group $3(P=0.001)$, Group 1, and Group $4(P=0.00)$ \& Group 2 and Group $4(P=0.047)$. Most of the subjects with the moderate handicap score on tinnitus handicap inventory fell in the Group 4 (56-65years age), however a large number of mild scoring subjects were found in the other three groups, i.e., Group 1, 2 and 3. It means that the tinnitus handicap increases as the age of the patient increases (Table 4).

Perceived stress scale was used for comparison of distress among four groups, likewise to THI scores, and the scores were again significantly different between the younger group (Group 1, 15-25years) and the other three groups. The significant $p$ values were observed between Group 1 and Group $2(P=0.014)$, Group 1 and Group 3 $(P=0.009)$, Group 1 and Group $4(P=0.006)$, respectively. The scores of Group 4 were falling in the very high distress range; however, the Group 1 scores mainly settled in low distress range. Moreover, the comparison of stress scores of Groups 2,3 and 4 did not show any significant difference (Table 5). Lastly, the severity of tinnitus between different age groups was compared using the Tinnitus Severity Index. The significant difference was

Table 1: Demographic variables.

\begin{tabular}{|c|c|c|c|c|c|}
\hline & & Group $1(\mathrm{~N}=15)$ & Group $2(N=15)$ & Group3(N=15) & Group $4(N=15)$ \\
\hline Age range (years) & & $15-25$ & $26-40$ & $41-55$ & $56-65$ \\
\hline Mean age(S.D.) & & $20.56(9.73)$ & $38.57(10.61)$ & $41.25(8.40)$ & $63.02(10.11)$ \\
\hline \multirow[t]{2}{*}{ Sex } & Male & $9(60 \%)$ & $10(66.7 \%)$ & $12(80 \%)$ & $6(40 \%)$ \\
\hline & Female & $6(40 \%)$ & $5(33.3 \%)$ & $3(20 \%)$ & $9(60 \%)$ \\
\hline \multirow[t]{2}{*}{ Tinnitus duration months } & $<12$ & $10(66.7 \%)$ & $11(73.3 \%)$ & $11(73.3 \%)$ & $1(6.67 \%)$ \\
\hline & $>12$ & $5(33.4 \%)$ & $4(26.7 \%)$ & $4(26.7 \%)$ & $14(93.3 \%)$ \\
\hline \multirow[t]{2}{*}{ Tinnitus localisation } & Right & $5(33.3 \%)$ & $4(26.7 \%)$ & $3(20 \%)$ & $3(20 \%)$ \\
\hline & Left & $10(66.7 \%)$ & $11(73.3 \%)$ & $12(80 \%)$ & $12(80 \%)$ \\
\hline \multirow[t]{2}{*}{ Tinnitus type } & Tonal & $2(13.3 \%)$ & $11(73.3 \%)$ & $10(66.7 \%)$ & $14(93.3 \%)$ \\
\hline & Noise & $13(86.6 \%)$ & $4(26.7 \%)$ & $4(26.7 \%)$ & $1(6.67 \%)$ \\
\hline
\end{tabular}

Table 2: Comparison of various tinnitus parameters among all the four groups.

\begin{tabular}{ccccccccccc} 
Domain & \multicolumn{2}{c}{ Group 1 } & \multicolumn{2}{c}{ Group 2 } & \multicolumn{2}{c}{ Group3 } & \multicolumn{2}{c}{ Group4 } & f & Significance \\
& Mean & S.D & Mean & S.D & Mean & S.D & Mean & S.D & & \\
Pitch of & 4672.14 & 4055.6 & 6394.2 & 4438 & 7065.2 & 3348.74 & 8608.3 & 2657.2 & 1.91 & \\
tinnitus & & & & & & & & & & \\
Intensity & 55.71 & 11.7 & 54.5 & 13 & 52.5 & 22.17 & 74.17 & 12.4 & 2.02 & 0.142 \\
THI & 20.14 & 9.38 & 32.29 & 14 & 36.27 & 6.81 & 41.33 & 14.56 & 6.58 & $\mathbf{0 . 0 0 1}$ \\
PSS & 11.01 & 4.08 & 20.41 & 11.59 & 20.17 & 6.74 & 21.34 & 5.39 & 3.32 & $\mathbf{0 . 0 2 8}$ \\
TSI & 12.03 & 0.57 & 15.13 & 1.72 & 14.75 & 1.44 & 16.113 & 1.16 & 13.62 & $\mathbf{0 . 0 0 1}$
\end{tabular}

p-value $<0.01=$ highly significant; ${ }^{\star} \mathrm{p}$-value $<0.05=$ significant; $\mathrm{p}$-value $>0.05=$ not significant (NS), THI-tinnitus handicap inventory, PSS -perceived stress scale, TSI - tinnitus severity index

Table 3: Multiple comparison of Tinnitus frequency among groups.

$\begin{array}{llccc}\text { Parameter } & \text { Comparison } & \text { Mean difference } & \text { Std. Error } & \text { P } \\ \text { Tinnitus Frequency } & \text { Group1 Vs Group 2 } & -1722.057 & 1753.255 & .331 \\ \text { Tinnitus Frequency } & \text { Group 1 Vs Group 3 } & -2393.079 & 1584.727 & .138 \\ \text { Tinnitus Frequency } & \text { Group 1 Vs Group 4 } & -3936.190^{*} & 1692.025 & * .025 \\ \text { Tinnitus Frequency } & \text { Group 2 Vs Group 3 } & -671.022 & 1403.177 & .635 \\ \text { Tinnitus Frequency } & \text { Group 2 Vs Group 4 } & -2214.133 & 1523.317 & .153 \\ \text { Tinnitus Frequency } & \text { Group 3 Vs Group 4 } & -1543.111 & 1325.877 & .251\end{array}$


Table 4: Multiple comparisons of tinnitus handicap inventory among four groups.

\begin{tabular}{|c|c|c|c|c|}
\hline Parameter & Comparison & Mean difference & Std. Error & $p$ \\
\hline $\mathrm{TH} I^{*}$ & Group1 Vs Group 2 & $-12.157^{\star}$ & 5.089 & .021 \\
\hline THI & Group 1 Vs Group 3 & $-16.135^{\star}$ & 4.600 & .001 \\
\hline THI & Group 1 Vs Group 4 & $-21.190 *$ & 4.911 & .000 \\
\hline THI & Group 2 Vs Group 3 & -3.978 & 4.073 & .334 \\
\hline THI & Group 2 Vs Group 4 & $-9.033^{\star}$ & 4.421 & .047 \\
\hline THI & Group 3 Vs Group 4 & -5.056 & 3.848 & .196 \\
\hline
\end{tabular}

Table 5: Multiple comparisons of Perceived stress scale between the four groups.

\begin{tabular}{ccccc}
\hline Parameter & Comparison & Mean diff & Std. Error & P \\
PSS* & Group1 Vs Group 2 & $-9.400^{*}$ & 3.681 & .014 \\
PSS & Group 1 Vs Group 3 & $-9.167^{*}$ & 3.327 & .009 \\
PSS & Group 1 Vs Group 4 & $-10.333^{*}$ & 3.553 & .006 \\
PSS & Group 2 Vs Group 3 & 0.233 & 2.946 & .937 \\
PSS & Group 2 Vs Group 4 & -.933 & 3.199 & .772 \\
PSS & Group 3 Vs Group 4 & -1.167 & 2.784 & .677 \\
${ }^{* *}$ p-value $<0.01$ = highly significant; * p-value<0.05= significant; p-value $>0.05=$ not significant (NS), PSS* - perceived stress scale
\end{tabular}

Table 6: Multiple comparisons on Tinnitus severity index among the four groups

\begin{tabular}{|c|c|c|c|c|}
\hline Parameter & Comparison & Mean diff & Std. Error & $\mathrm{P}$ \\
\hline TSI* & Group1 Vs Group 2 & $-3.100 *$ & .670 & .000 \\
\hline TSI & Group 1 Vs Group 3 & $-2.722^{\star}$ & .605 & .000 \\
\hline TSI & Group 1 Vs Group 4 & $-4.083^{\star}$ & .646 & .000 \\
\hline TSI & Group 2 Vs Group 3 & .378 & .536 & .485 \\
\hline TSI & Group 2 Vs Group 4 & -.983 & .582 & .098 \\
\hline TSI & Group 3 Vs Group 4 & $-1.361^{\star}$ & .507 & .010 \\
\hline
\end{tabular}

chiefly observed between Group 1 and the other three groups. Group 1 perceived tinnitus severity in very mild range, in comparison to the other three groups essentially falling in mild category. In conducting Post hoc analysis significant, differences were observed between Group 1 and Group $2(P=0.000)$, Group 1 and Group $3(P=0.00)$, Group 1 and Group $4(P=0.00)$, Group 3 and Group 4 $(P=0.010)$.

\section{DISCUSSION}

Tinnitus prevalence has been many times associated with senescence as well as concomitant hearing loss, 11. However, our study emphasizes "age" solely as a critical contributing factor towards the distress, handicap, and severity of tinnitus. Recent research revealed that subjects above 40 years of age were essentially affected with severe tinnitus, moreover the severity of stress was far greater in these patients, ${ }^{16}$. Literature also stated that subjects above 40 years of age are less able to deal with tinnitus and thus have higher depression scores 3 . The present study also supports the research stating that "patient's reaction to tinnitus is a complex interaction between acoustic phantom symptoms, somatic attention, and depressive symptoms", 17. Most of the subjects in group 4 (56-65 years) had a higher score of distress, and severity, and tinnitus handicap, thus increasing their risk for depression \& anxiety. This could be attributed to the steady worsening of the tinnitus over a passage of time and the tendency of subjects to tolerate the symptoms of non- life-threatening conditions like tinnitus,16. Similar results have also been reported by Swiahb JA et al. that tinnitus largely affects individuals 40-60yrs of age, followed by above 60 years of age, \& then less than 40 years of age. They supported their finding of the largest tinnitus prevalence in $40-60 y e a r s$ of subjects, as $67.7 \%$ of these subjects present with sensorineural hearing loss ${ }^{16}$.

The location of tinnitus, the type of tinnitus, was similar to what has been reported by previous researchers, 17,18. The left ear is more susceptible to injury and prone to tinnitus due to the anatomical variations in the efferent system of the left and right side ${ }^{19,20}$. The younger age group reported with more noise-like tinnitus \& adult and older subjects with more tonal tinnitus. Previous researchers have also reported $2718 \mathrm{~Hz}$ to be the average value of tinnitus in adults ${ }^{21}$. Some researchers reported that the tinnitus frequency concentrated beyond $2000 \mathrm{hz}$ and largely above $4000 \mathrm{~Hz}{ }^{22,23}$. Tinnitus handicap inventory measures the impact of tinnitus on daily life ${ }^{24}$; however tinnitus severity index studies the emotional and psychological impact of tinnitus on the subject's life and perceived stress scale measures the perception of stress. In the present study, subjects between 56-65years of age showed a significantly larger impact of tinnitus on daily life as well as the stress, emotional and psychological 
wellbeing, \& least impact of tinnitus noticed in the youngest group (15-25years). Another researcher in 2009 documented that men and senior citizens reported with loud and annoying tinnitus, ${ }^{25}$. Axelson and Ringdehl further revealed that greater the tinnitus severity, chances of sleep disturbances increase among the tinnitus subjects, ${ }^{26}$. It can thus be hypothesized that subjects in the age range of 56-65yrs were more emotionally aroused and made greater attempts to cope with their tinnitus and therefore reported with a greater number of problems and emotional focused coping behaviour ${ }^{27}$. Thus, the results of the present study provide significant information to audiologists while intervening geriatric subjects suffering from tinnitus because these subjects certainly require modifications in the diagnostic as well as tailor-made intervention strategies ${ }^{28}$

\section{CONCLUSION}

This study showed that increasing age not only led to changes in tinnitus frequency, but also increased severity and handicap. In the younger population tinnitus was less stressful and had a limited effect on their daily life. The gradual increase in tinnitus severity could be attributed to the deterioration of brain mechanics due to aging.

\section{REFERENCES}

1. Jastreboff PJ. Phantom auditory perception (tinnitus): mechanisms of generation and perception. Neuroscience Research. 1990:221-54.

2. Jastreboff PJ, Jastreboff MM. Tinnitus Retraining Therapy (TRT) as a Method for Treatment of Tinnitus and Hyperacusis Patients. J Am Acad Audiol. 2000.

3. Jastreboff PJ, Gray WC, Gold SL. Neurophysiological approach to tinnitus patients. Am $\mathrm{J}$ Otol [Internet]. 1996;17(2):236-40.

4. Yenigün A, Doğan R, Aksoy F, Akyüz S, Dabak H. Assessment of tinnitus with tinnitus severity index, tinnitus handicap inventory and distortion product otoacoustic emissions in patients with normal hearing and hearing loss. Kulak Burun Bogaz Ihtis Derg. 2014;1;24(1):11-6.

5. Shargorodsky J, Curhan SG, Curhan GC, Eavey R. Change in prevalence of hearing loss in US adolescents. JAMA - J Am Med Assoc. 2010;18;304(7):772-8.

6. Li CM, Zhang X, Hoffman HJ, Cotch MF, Themann CL, Wilson MR. Hearing Impairment Associated With Depression in US Adults, National Health and Nutrition Examination Survey 2005-2010. JAMA Otolaryngol Neck Surg. 2014;1:4-12.

7. Eggermont JJ, Roberts LE. The neuroscience of tinnitus. Trends Neurosci. 2004;27(11):676-82.

8. Adrian D, Refaie A El. The epidemiology of tinnitus. Singular; 2000:1-23.

9. Stouffer JL, Tyler RS. Characterization of tinnitus by tinnitus patients. J Speech Hear Disord. 1990;55(3):439-53.

10. Lockwood AH. Tinnitus. A Neurologic Clinics. 2005:893-900.

11. Meikle M, Taylor-Walsh E. Characteristics of tinnitus and related observations in over 1800 tinnitus clinic patients. $J$ Laryngol Otol Suppl [Internet]. 1984;9:17-21.
12. Folmer RL, Griest SE, Meikle MB, Martin WH. Tinnitus severity, loudness, and depression. Otolaryngol Neck Surg [Internet]. 1999;121(1):48-51.

13. Newman CW, Jacobson GP, Spitzer JB. Development of the tinnitus handicap inventory. Arch Otolaryngol - Head Neck Surg. 1996;122(2):143-8.

14. Meikle MB, Griest SE S t B. Press LS. Measuring the negative impact of tinnitus: $A$ brief severity index. In: Ryan $A$, editor. Abstr Eighteenth Midwinter Res Meet Assoc Res Otolaryngol Des Moines, IA Assoc Res Otolaryngol. 1995;167:1-4.

15. Solis J, Cohen S, Kamarck T, Mermelstein R. A GLOBAL MEASURE OF PERCEIVED STRESS. Vol. 24, Journal of Health and Social Behavior. 1983.

16. Al-Swiahb J, Park SN. Characterization of tinnitus in different age groups: A retrospective review. Noise Heal. 2016;1:18(83):214-9.

17. McCombe A, Baguley D, Coles R, McKenna L, McKinney C, Windle-Taylor $P$. Guidelines for the grading of tinnitus severity: The results of a working group commissioned by the British Association of Otolaryngologists, Head and Neck Surgeons, 1999. Clin Otolaryngol Allied Sci. 2001;26(5):388-93.

18. Coad M Lou, Lockwood A, Salvi R, Burkard R. Characteristics of patients with gaze-evoked tinnitus. Otol Neurotol. 2001;22(5):650-4.

19. Epstein LJ, Kristo D, Strollo PJ, Friedman N, Malhotra A, Patil $\mathrm{SP}$, et al. Clinical guideline for the evaluation, management and long-term care of obstructive sleep apnea in adults. J Clin Sleep Med [Internet]. 2009;3:263-76.

20. Schmidt CM, Knief A, Lagosch AK, Deuster D, Am ZehnhoffDinnesen A. Left-right asymmetry in hearing loss following cisplatin therapy in children - The left ear is slightly but significantly more affected. Ear Hear. 2008 Dec;29(6):830-7.

21. Briner W, House J, O'Leary M. Synthetic Prostaglandin E1 Misoprostol as a Treatment for Tinnitus. Arch Otolaryngol Head Neck Surg [Internet]. 1993;119(6):652-4.

22. Akkuzu B, Yilmaz I, Cakmak O, Ozluoglu LN. Efficacy of misoprostol in the treatment of tinnitus in patients with diabetes and/or hypertension. Auris Nasus Larynx [Internet]. 2004;31(3):226-32.

23. Yilmaz I, Akkuzu B, Çakmak Ö, Özlüoglu LN. Misoprostol in the Treatment of Tinnitus: A Double-Blind Study. Otolaryngol Neck Surg [Internet]. 2004;130(5):604-10.

24. Heller AJ. Classification and epidemiology of tinnitus. Otolaryngol Clin North Am . 2018;17:239-48.

25. Goebel G, Roseneck SK. When Tinnitus Loudness and Annoyance Are Discrepant: Audiological Characteristics and Psychological Profile. 2007

26. Axelsson A, Ringdahl A. Tinnitus-A study of its prevalence and characteristics. Br J Audiol. 1989;23(1):53-62.

27. Dineen R, Doyle J, Bench J. Audiological and psychological characteristics of a group of tinnitus sufferers, prior to tinnitus management training. Br J Audiol. 1997;31(1):27-38.

28. Fagelson MA. The association between tinnitus and posttraumatic stress disorder. Am J Audiol. 2007 Dec;16(2):107-17. 